\title{
Effectiveness of Somatic Balance Restoration Therapy to Alleviate Pain of Musculoskeletal System
}

\author{
Munehiro Mike Kayo, Yoshiaki Ohkami \\ Institute of System Design and Management, Yokohama, Japan \\ Email:kayo.mike@hotmail.com,ohkami@sdm.keio.ac.jp
}

How to cite this paper: Kayo, M.M. and Ohkami, Y. (2017) Effectiveness of Somatic Balance Restoration Therapy to Alleviate Pain of Musculoskeletal System. Health, 9, 1390-1403.

https://doi.org/10.4236/health.2017.910102

Received: April 29, 2017

Accepted: September 22, 2017

Published: September 25, 2017

Copyright ( $\odot 2017$ by authors and Scientific Research Publishing Inc. This work is licensed under the Creative Commons Attribution International License (CC BY 4.0).

http://creativecommons.org/licenses/by/4.0/

\section{(c) (i) Open Access}

\begin{abstract}
Chronic pain and general physical discomfort are common symptoms among those seeking medical or physiotherapy treatment, as it relates to disorders found in the Human Musculoskeletal System (HMS). Since this system is highly complex and large in scale, clinical pain research has been confounded by many complex factors. The goal of our research is to overcome these obstacles by applying multidisciplinary approaches including systems engineering, traditional oriental techniques, conventional medicine and related sciences. To pursue such an integrated approach this paper examines the therapist-guided exercise for restoring human musculoskeletal balance called the Somatic Balance Restoration Therapy (SBRT). The SBRT is a simple but effective self-exercise therapy with minimal assistance by a trained therapist. This therapy is analyzed by a mechanical engineering method by modeling the human body as a multi-body subject to a static equilibrium condition. In addition, the wording has been rewritten in functional anatomical terms, enabling smooth communication between specialists of three different disciplines: therapy, conventional medicine and systems engineering. Examples will be given to demonstrate an integrated and systematic approach for identifying and remedying malfunctions within the HMS.
\end{abstract}

\section{Keywords}

Integrated Medicine, Self-Exercise Therapy, Musculoskeletal Pain, Systems Approach

\section{Introduction}

Chronic pain and general physical discomfort can be attributed to disorders or 
malfunctions within the Human Musculoskeletal System (HMS). To help alleviate these symptoms, Asian countries such as Japan and China have developed various traditional medicine-based exercise techniques over many years [1]. However, some of reviewers are critical to such non-orthodox medicine [2] [3] [4] but some others support by stating "overwhelming effort toward attempts at integrating alternative medicine into mainstream" [5]. In addition, these techniques have been enhanced by recent scientific research into the HMS, undertaken by biomedical and mechanical engineers.

The objective of this paper is to demonstrate the effectiveness of a practice of the clinical technique named Somatic Balance Restoration Therapy (SBRT) by applying mechanical and systems engineering methods. The SBRT is a therapist-guided self-exercise technique that helps the patient to perform easily a series of simple motions in a completely non-invasive manner. By applying a systems and mechanical engineering approach, a computerized visualization of the SBRT's clinical technique has been demonstrated. This process was then evaluated by applying matrix algebra and correlation analysis. For the purpose of integration into conventional medicine, the terminology used by the therapist (one of the authors of this paper) is converted to the functional anatomical terms. This effort has turned out that such wording coincides with the mechanical symbols of robotic systems. Next, the evaluation was applied to a typical actual therapy records and the results revealed that the systems approach developed herein was proven valuable and gives scientific background of complementary medicine.

\section{Previous Works}

Chronic pain and general physical discomfort are common symptoms. However, these conditions convey important information on the clinically relevant state of the human body, especially relating to disorders or malfunctions found within the Human Musculoskeletal System (HMS) [6]. For many years, acute and chronical pain issues have been reported in various forms or symptoms: neck and extremity disorders among computer users, knee or hip complaints in senior adults [7], and recovery after minor traffic accident injuries. Most pain research works have been conducted in Western countries such as the United States, Germany, Denmark, Sweden, France and others [8] [9]. Worldwide, however, millions of people suffer from untreated pain, particularly in the developing world where the burden is highest among the poor. Reducing global inequalities in untreated pain requires a concerted effort by global health funders, institutions, and organizations. These groups must overcome the complexity of pain management by promoting multidisciplinary and holistic approaches that integrate traditional oriental techniques with Western medicine. In China and Japan, pain is widely treated by therapeutic approaches by correcting HMS distortions. Most of the above-cited studies are based on statistical analysis with a large number of samples. Lars and Swenson [6] conducted an overall survey on 
clinical findings of referred muscle pain, while Feine and Lund [9] assessed the efficacy of physical therapy in controlling chronic musculoskeletal disorders. In addition, a systematic review of exercise therapies that help in overcoming chronic lower back pain has been compiled. Exercise therapy has recently been recognized as part of Integrated Medicine as shown in Figure 1. However, clinical pain research is confounded by many factors that obscure specific aspects of the underlying disease, such as biases in the cognitive, emotional, and social aspects of the ailment. Moreover, pain is a multidimensional and highly individualized perception that is difficult to quantify or validate. Apart from such medical and psychological aspects of pain in the HMS, engineering approaches have been developed especially by applying mechanical engineering of the HMS methods, where anatomical motions are expressed by using mechanical element motions such as joint rotation [10] [11] [12]. However, actual motions of the real HMS are not fully related to engineering methods or tools.

In order to overcome such difficulties, this paper aims to realize a systematic interpretation of a therapist-guided technique called the Somatic Balance Restoration Therapy (SBRT) [13] [14] [15]. The methodologies used are based on mechanical and systems engineering applied to a number of therapy data recorded by one of the authors. By applying this approach the SBRT emerges as an academically appropriate technique representing a step forward in exercise therapy. With clear visualization of the method, this technique can be used as an educational tool for the inexperienced practitioner and as a management tool for established practitioners.

\section{Methods}

\subsection{Therapist-Guided Exercise: Somatic Balance Restoration Therapy}

Figure 2 shows a flow diagram of the SBRT system approach which begins with a "Therapist-Guided Motion Test". During this test, the patient lays in a face up (supine) or face down (prone) position and performs a series of guided body movements called Active Motions shown in Figure 3 and Table 1. There are basically 40 active motions with left and right directions, that makes 80 motions altogether. The therapist systematically guides the patient to try each of the active motions from \#1 to \#80. It is important to tell the patient to perform it only if it is easy, and not to perform the motion if the patience feels pain or hard to do so. In short, the therapist will attempt to guide the patient through each Active

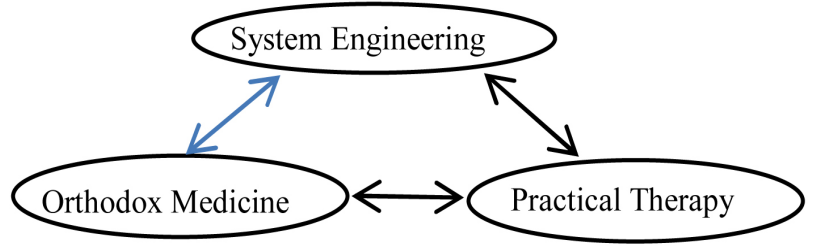

Figure 1. Integrated medicine scheme. 


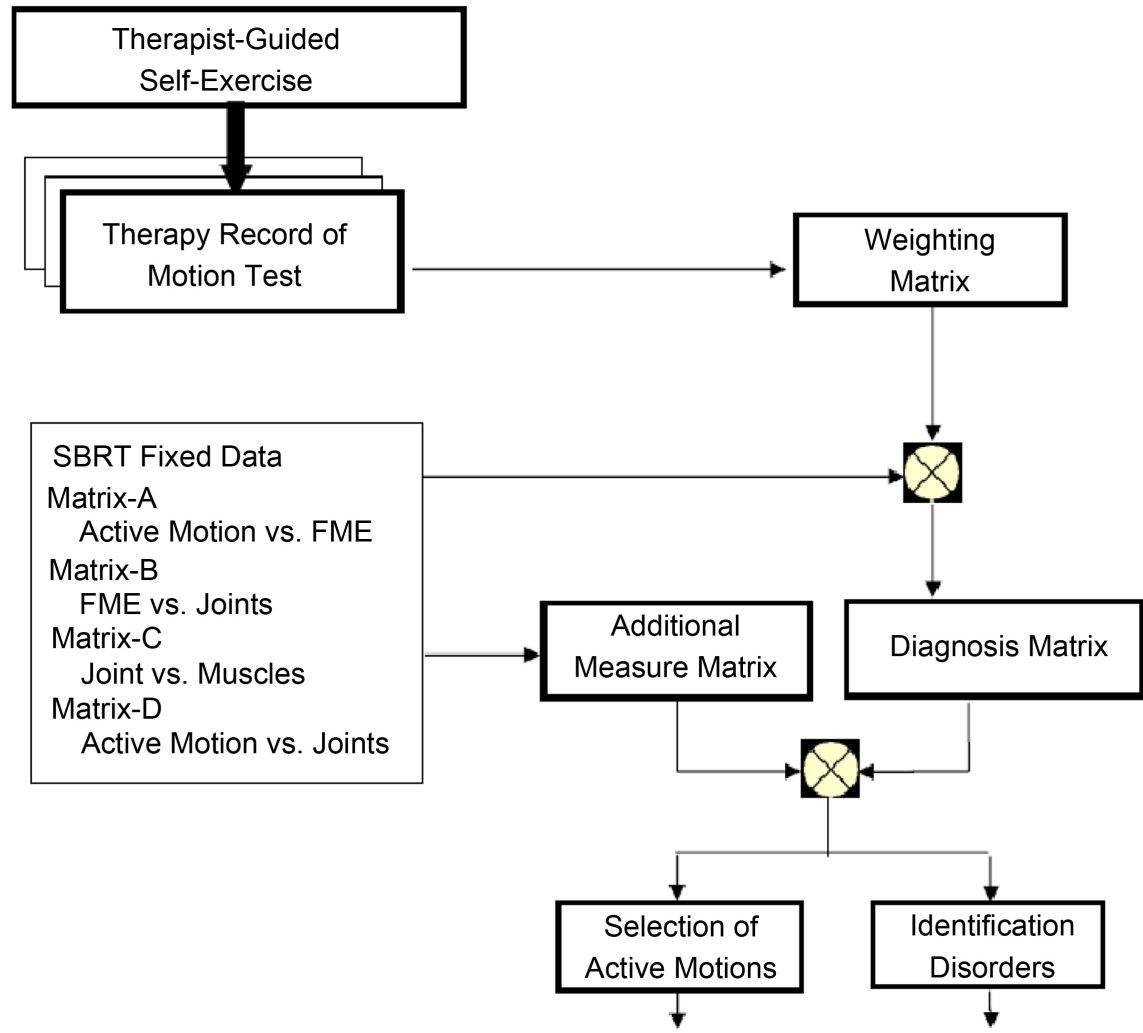

Figure 2. Flow diagram of the SBRT process.

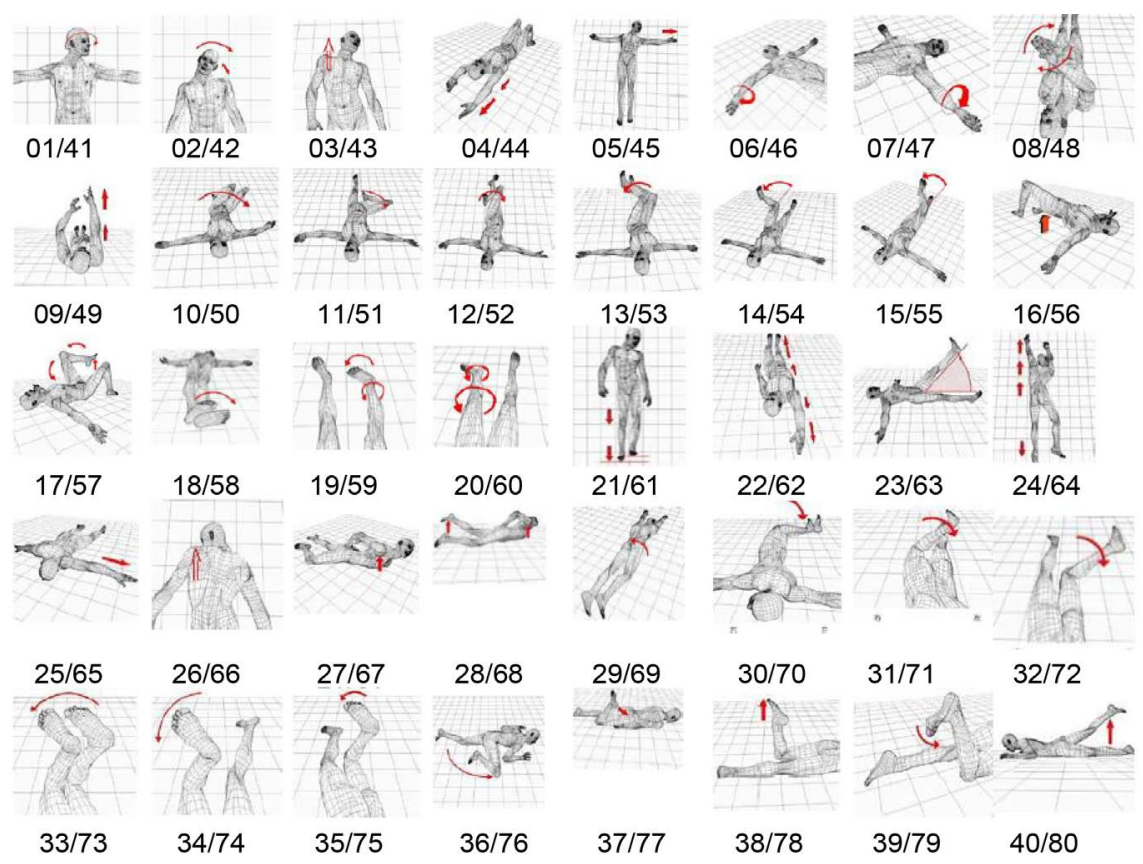

Figure 3. Eighty active motions of SBRT.

Motion (patient initiated motion) listed in Table 1 and to record the results in the SBRT sheet. It is noted that Motions from \#81 to \#138 in Table 2 (to be called Associate Motions and not performed intentionally by the patient) are 
Table 1. List of active motion.

\begin{tabular}{|c|c|c|c|}
\hline No. & Motion & No. & Motion \\
\hline 1 & Turn neck toward left & 41 & Turn neck toward right \\
\hline 2 & Tilt head toward left & 42 & Tilt head toward right \\
\hline 3 & Elevate right shoulder to head & 43 & $\begin{array}{l}\text { Elevate left shoulder to head } \\
\text { toward head }\end{array}$ \\
\hline 4 & Stretch right arm above head & 44 & $\begin{array}{c}\text { Stretch left arm above } \\
\text { head }\end{array}$ \\
\hline 5 & Extend arm to left & 45 & Extend arm to right \\
\hline 6 & Down rotation of right arm & 46 & Up rotation of right arm \\
\hline 7 & Upward rotation of left arm & 47 & Downward rotation of left arm \\
\hline 8 & Twist both arms toward left & 48 & Twist both arms toward right \\
\hline 9 & Stretch right arm upward & 49 & Stretch left arm upward \\
\hline 10 & Swing both knees toward right & 50 & Swing both knees toward left \\
\hline 11 & Swing right knee outward & 51 & Swing right knee inward \\
\hline 12 & Swing left knee inward & 52 & Swing left knee outward \\
\hline 13 & Swing both legs toward left & 53 & Swing both legs toward right \\
\hline 14 & Swing right lower leg inward & 54 & Swing right lower leg outward \\
\hline 15 & Swing left lower leg outward & 55 & Swing left lower leg inward \\
\hline 16 & Elevate left hip upward & 56 & Elevate right hip upward \\
\hline 17 & Raise left knee & 57 & Raise right knee \\
\hline 18 & Twist both legs to left & 58 & Twist both legs to right \\
\hline 19 & Rotate right leg inward & 59 & Rotate right leg outward \\
\hline 20 & Rotate left leg outward & 60 & Rotate left leg inward \\
\hline 21 & Stretch right heel & 61 & Stretch left heel \\
\hline 22 & Stretch right arm and heel & 62 & Stretch left arm and heel \\
\hline 23 & Raise left leg off ground & 63 & Raise right leg off ground \\
\hline 24 & Stretch left arm above head & 64 & Stretch right arm above head \\
\hline 25 & Extend left arm to left & 65 & Extend right arm to right \\
\hline 26 & Elevate left shoulder upward & 66 & Elevate right shoulder upward \\
\hline 27 & Twist left shoulder off ground & 67 & Twist right shoulder off ground \\
\hline 28 & Raise right shoulder \& left leg & 68 & Raise left shoulder \& right leg \\
\hline 29 & Twist right hip off ground & 69 & Twist left hip off ground \\
\hline 30 & Swing both knees toward left & 70 & Swing both knees toward right \\
\hline 31 & Swing left knee inward & 71 & Swing right knee inward \\
\hline 32 & Swing left knee outward & 72 & Swing right knee outward \\
\hline 33 & Rotate both legs to right & 73 & Rotate both legs to left \\
\hline 34 & Rotate right leg outward & 74 & Rotate right leg inward \\
\hline 35 & Rotate left leg inward & 75 & Rotate left leg outward \\
\hline 36 & Raise right knee to shoulder & 76 & Raise left knee to shoulder \\
\hline 37 & Pull right heel toward hip & 77 & Pull left heel toward hip \\
\hline 38 & Push left foot upward & 78 & Push left foot downward \\
\hline 39 & Pull right foot downward & 79 & Pull right foot upward \\
\hline 40 & Raise right leg off ground & 80 & Raise left leg off ground \\
\hline
\end{tabular}


Table 2. List of associated motion.

\begin{tabular}{|c|c|c|c|}
\hline No. & Motion & No. & Motion \\
\hline 81 & Turn neck toward left & 110 & Turn neck to right \\
\hline 82 & Elevate right shoulder to head & 111 & Elevate left shoulder to head \\
\hline 83 & Raise right shoulder off ground & 112 & Raise left shoulder off ground \\
\hline 84 & De-elevate left shoulder from head & 113 & De-elevate right shoulder from head \\
\hline 85 & Push left shoulder against ground & 114 & Push right shoulder against ground \\
\hline 86 & Pull right arm to body & 115 & Pull left arm to body \\
\hline 87 & Push right hip bone to ground & 116 & Push left hip bone to ground \\
\hline 88 & Contract left hip bone to head & 117 & Contract right hip bone to head \\
\hline 89 & Elevate left hip bone off ground & 118 & Elevate right hipbone off ground \\
\hline 90 & Close right leg inward & 119 & Close left leg inward \\
\hline 91 & Push left leg outward & 120 & Push right leg outward \\
\hline 92 & Pull left knee to head & 121 & Pull right knee to head \\
\hline 93 & Bend left knee up off ground & 122 & Bend right knee up off ground \\
\hline 94 & Bend left knee up off ground & 123 & Stretch left heel downward \\
\hline 95 & Contract left heel to head & 124 & Contract right heel to head \\
\hline 96 & Turn neck to right & 125 & Turn neck to left \\
\hline 97 & De-elevate right shoulder from head & 126 & De-elevate left shoulder from head \\
\hline 98 & Push left shoulder against ground & 127 & Push right shoulder against ground \\
\hline 99 & Pull right arm to body & 128 & Pull left arm to body \\
\hline 100 & Contract right arm to feet & 129 & Contract left arm to feet \\
\hline 101 & Contract right hip bone to head & 130 & Contract left hipbone to head \\
\hline 102 & Elevate right hip bone off ground & 131 & Elevate left hipbone off ground \\
\hline 103 & Push left hip bone to ground & 132 & Push right hipbone to ground \\
\hline 104 & Push left leg outward & 133 & Push right leg outward \\
\hline 105 & Pull right knee to head & 134 & Push right leg outward \\
\hline 106 & Contract right heel to head & 135 & Contract left heel to head \\
\hline 107 & Push left knee against ground & 136 & Contract left heel to head \\
\hline 108 & Raise left heel off ground & 137 & Raise right heel off ground \\
\hline 109 & Push left knee against ground & 138 & Push right knee against ground \\
\hline
\end{tabular}

induced only by the Active Motions from \#1 to \#80 in Table 1 . Thus, the whole Active and Associated Motions are called Fundamental Motion Elements (FME).

The goal of the SBRT is to eliminate or at least alleviate overall pain or discomfort, which includes any pain during the therapy. This is achieved by the patient reporting varying degrees of difficulty while performing the motion test. As mentioned, the therapist does not have physical contact with the patient during the examination or the guided self-exercise phase of the therapy. However, to assist the patient's performance of certain motions, the therapist may apply slight resistance against the patients' limbs while performing problematic motions. 


\subsection{Relation between Active Motions and Anatomical}

A patient can perform any of the Active Motions with intension to do it, but usually cannot perform a single anatomical motion.

For example, if the patient intends to extend arm to left (Active Motions \#5), it is realized by horizontal adduction and retraction of the shoulder together with flexion of the elbow and extension of the wrist. It is noted that the anatomical motions correspond to the joint degrees-of-freedom of 15 body human model of Figure 4 and its robotic symbol expression of Figure 5. Table 3 shows the relation between active motions and anatomical motions. Such relations are conveniently represented by matrix format to be developed in the following section.

\subsection{Matrix Representation of Interconnecting Motions}

Among the 138 FMEs, the 80 Active Motions are intentionally performed by the patient (Table 1); the remaining 58 are Associated Motions induced by Active Motions (Table 2). FMEs are numbered \#1 to \#80 for Active Motions (Table 1). Of the Associated Motions in Table 2, FMEs \#81-\#109 are induced while in the face up (supine) position, while FMEs \#110-138 are induced while in the face down (prone) position. Please note that although FMEs \#1-80 are typically Active Motions, they can also act as Associated Motions in certain instances.

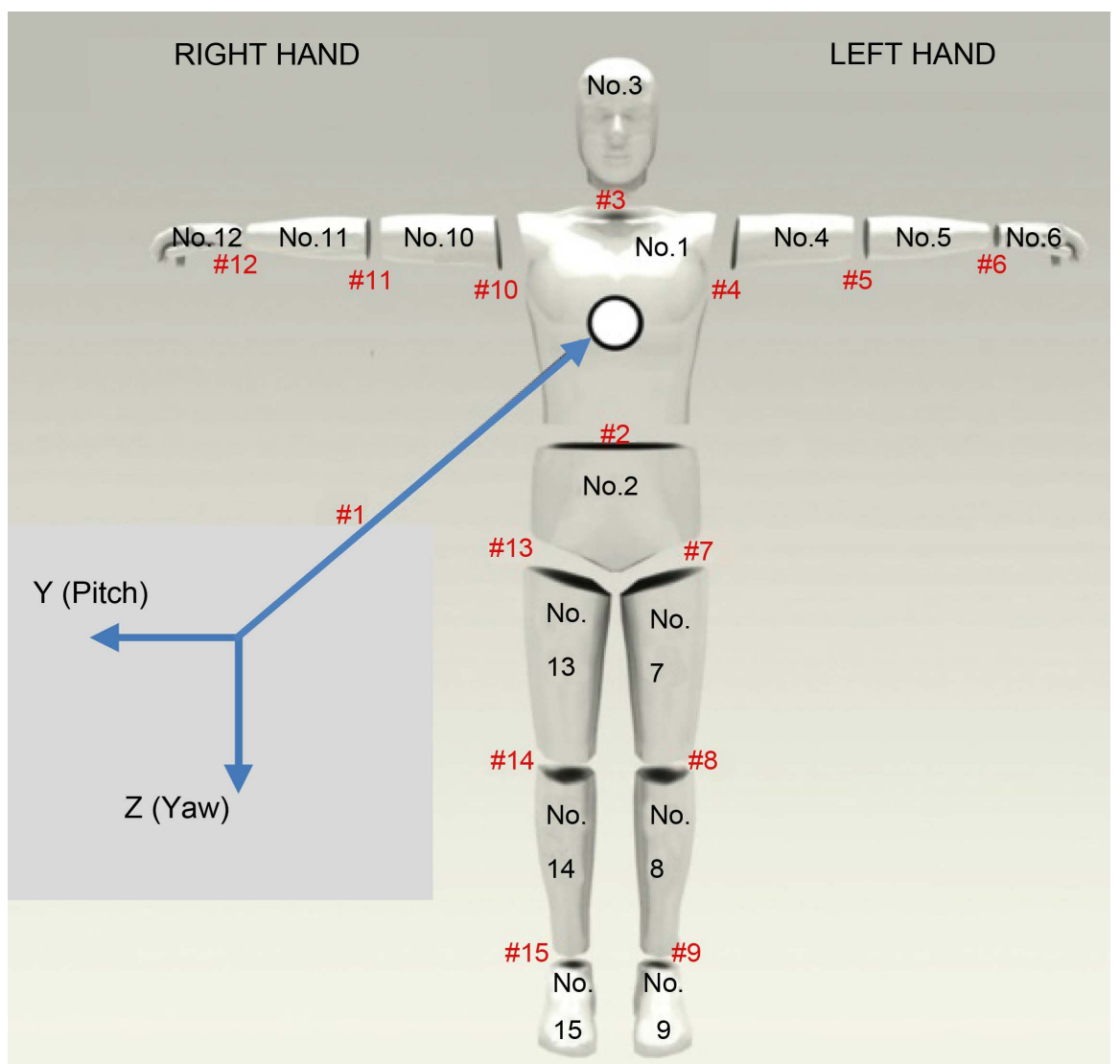

Figure 4. 15 body model. 


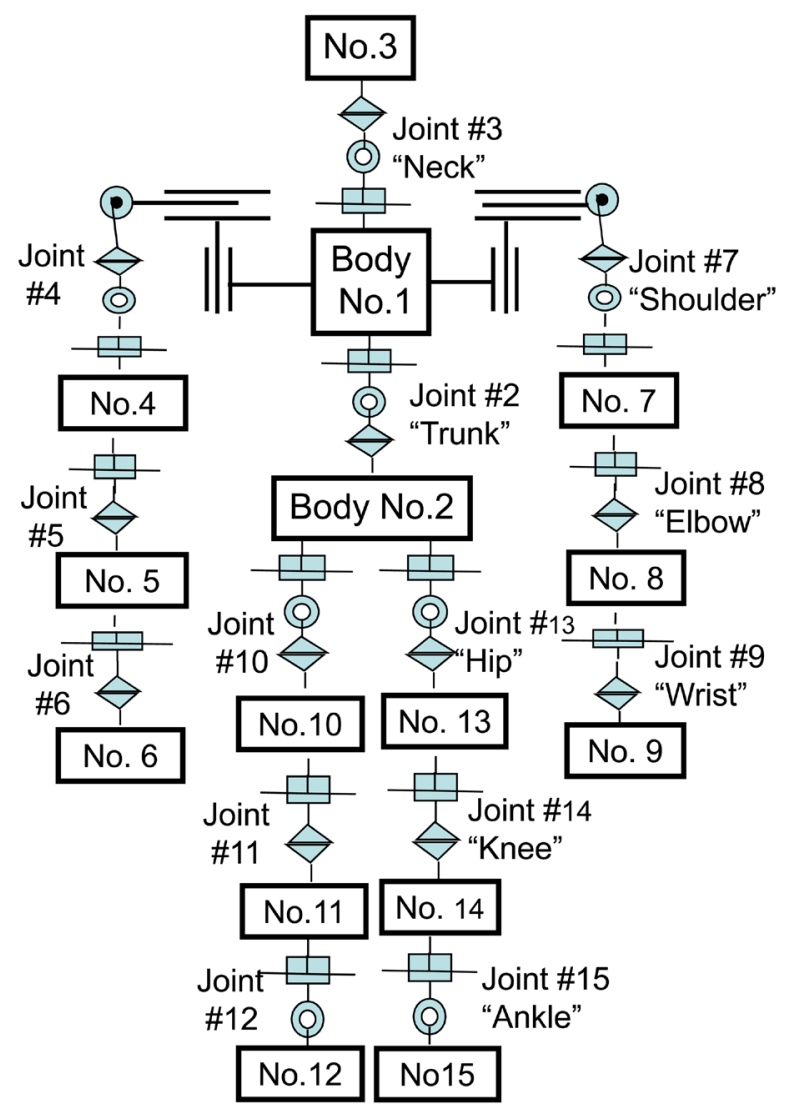

Figure 5. Motion diagram represented by JIS B0138.

Since the HMS is interconnected in a highly complex manner, each individual segment or component of it requires an integrative approach [13] [14] [15]. Active Motions are also interconnected with other Active and Associated Motions. Therefore, the relationship between any two of the 138 FME can be expressed by an $\mathrm{N}$-square matrix [13] of dimension 138. Likewise the relationship between Active Motions and Fundamental Motion Elements is represented by a 80 by 138 matrix to be called Matrix-A.

The above-mentioned processes are interpreted in terms of orthodox medicine as shown in Table 3. It is noted that an Active Motion induces one or more joint motions in most case, and human body motion can be realized in a combination of a few joint motions. Table 4 will be useful to bridge the gap between orthodox and practitioner medical approaches for dealing musculoskeletal aspects of the HMS. In order words, the 138 FMEs is related to some of the 80 joint motions that correspond to anatomical motions, and this relation is represented by a matrix of dimension 138 by 80 to be called Matrix-B.

\section{A Systems Approach to Identify Malfunctions and to Select Remedying Motions}

The SBRT process is digitized and visualized by extensive use of matrix representation, where the $(i, j)$ elements of Matrix A and B are denoted by $a(i, j)$ 
Table 3. Articulation names of joints in anatomical posture.

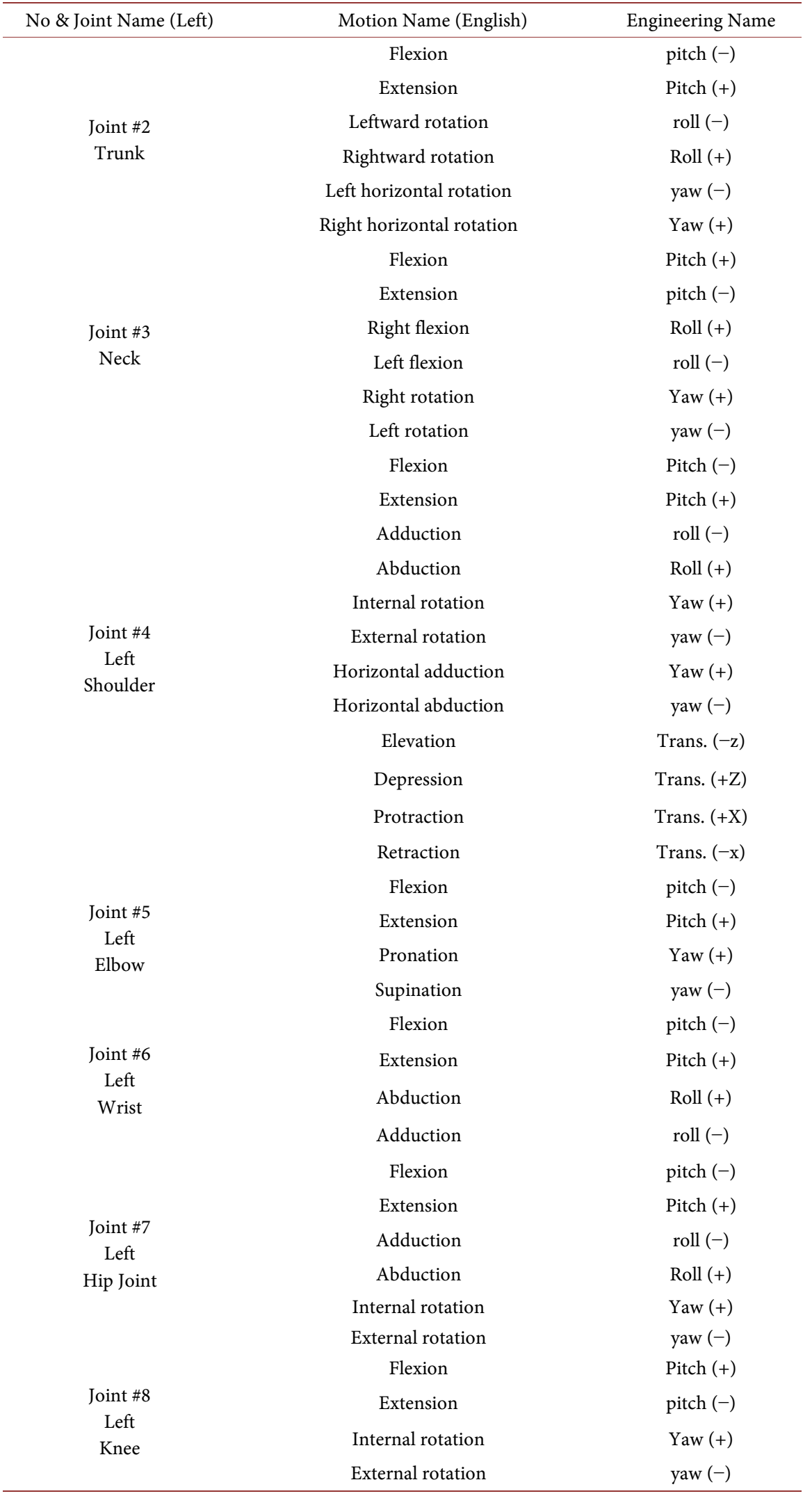

Note: Only left limb joints are cited. 
Table 4. An example of SBRT record (partially shown).

\begin{tabular}{ccc}
\hline No A0005_TN_0001 & \multicolumn{2}{c}{ No. N0005 } \\
\hline Motions & Check & Pain \\
\hline 41/01 Turn neck to (R/L) & e/p1 & B-2 \\
42/02 Tilt head toward (R/L) & e/p2 & B-2 \\
45/05 Extend arm to (R/L) & h/e & \\
11/51 Swing right knee outward (R/L) & e/p3 & G-8 \\
$53 / 13$ Swing both legs toward (R/L) & h/e & \\
$54 / 14$ Swing right lower leg inward (R/L) & e/p6 & F-1 \\
$55 / 15$ Swing left lower leg outward (R/L) & h/e & \\
$56 / 16$ Elevate hip upward (R/L) & e/p4 & G16, 17, 18 \\
\hline
\end{tabular}

Note: R/L $=$ Right/Left, $\mathrm{h}=$ hard, $\mathrm{e}=$ easy, na = not applied, B-2, F-1, F-5, G-8 etc: Body portions (See Figure 3).

and $b(i, j)$ respectively, then we can then we can define diagnostic matrices, $Q$ by

$$
Q=[q(i, k)]=\left[w_{i} d(i, k)\right] \quad(i=1, \cdots, 80 ; k=1, \cdots, 80)
$$

where

$$
d(i, k)=\sum_{j=1}^{136} a(i, j) \cdot b(j, k) \quad(i=1,80 ; j=1,80)
$$

The Active Motion during the Motion Test is weighted by quantity $w_{P}$ whose numerical value reflects the results (easy, hard or painful, denoted $e, h$ and $p$, respectively). The weighting values are determined subjectively yet skillfully by the therapist or the analyzer through experience. Note that easy movements are assigned large positive weightings, while painful movements are negatively weighted. We evaluate the selection of the Active Motion by computing correlation coefficients of the joint diagnosis matrix. The correlation coefficient is given by

$$
\operatorname{corr}(i, k)=\frac{\sum_{j=1}^{n}\left(x_{j}^{i}-\bar{x}^{i}\right)\left(y_{j}^{k}-\bar{y}^{k}\right)}{\sqrt{\sum_{j=1}^{n}\left(x_{j}^{i}-\bar{x}^{i}\right)} \sqrt{\sum_{j=1}^{n}\left(y_{j}^{i}-\bar{y}^{k}\right)}}
$$

where, in the joint diagnosis matrix of Equation (1),

$$
\begin{aligned}
x_{j}^{i} & =\text { pain part of }[q(i, j)], \\
y_{j}^{i} & =\text { easy part of }[q(i, j)]
\end{aligned}
$$

Traditionally, the SBRT therapist (and other Eastern medicine therapists) directly records patient examination results into a simple human-body model, and then identifies the malfunctioning point as the remedial exercise motion. Because expert therapists are relied on many years of personal experience and success to diagnose and treat patients, this knowledge was not wholly available to 
successors. This difficulty in transferring skills and experience to the next generation has motivated us to development a computer software support system that allows successors to learn from expert therapists and their experiences. The process of this therapeutic approach, as applied to actual cases is detailed below:

1) From the motion test results, identify the body motions that cause pain or discomfort

2) List the Active Motions associated with the pain-inflicting motion(s)

3) Identify the joint movements and muscle groups that are associated with the painful motions

4) Search for a frequency distribution pattern in the identified joint movements and muscle groups

5) Identify the joints and muscles associated with the highest frequency or reoccurrence of reported pain as the most probable causes of the malfunction(s)

6) From the identified rotary joints and muscle groups associated with the highest frequency of pain reports, find the Associated Motions using the charts of relationships between FMEs and Joint Motions

7) Identify the Active Motion that is interconnected with the Associated Motion, limiting the search to motions that can be comfortably performed by the patient

8) Identify the frequency distribution of the identified Active Motion

9) The Active Motion most frequently reported as comfortable will be applied as the remedial exercise motion in therapy

Note that Steps 1 - 5 identify candidate locations of the malfunction, while Steps 6 - 9 identify the corrective motions for therapy.

The Flow Diagram of Figure 2 is a visual representation of the computerized SBRT process. The inputs are therapeutic results constructed from the list of the pain-causative motions listed in Table 4. From these, the relationship(s) between pain-causative Active Motions and joint DOF are generated. The diagnostic matrix $Q$ is then determined by the identification of these relationships.

The results of the patient feeling, easy, hard or painful, are recorded on the SBRT sheet as illustrated by an example shown in Table 4. In this example, the patience can perform Motion \#41 (turning neck to right) easily, but cannot do Motion \#1(turn neck to left), because the patient feels pain at a specific location B-2 in Figure 6 and Figure 7.

Through the proposed process we can identify comfortable motions that induce joint motions consequent to Associated Motions. The goal of the SBRT is to find comfortable Active Motions that activate Joint Motions reported as painful. However, in general, these motions are not uniquely specified and thus the therapist must select the most effective motions among multiple comfortable Active Motions. For this purpose, we apply the cross-correlation analysis together with the "Distance Measure", defined as the number of joints between the malfunctioning zone and the motion-related joint.

The objective is to select or specify the Active Motion(s) that most effectively 

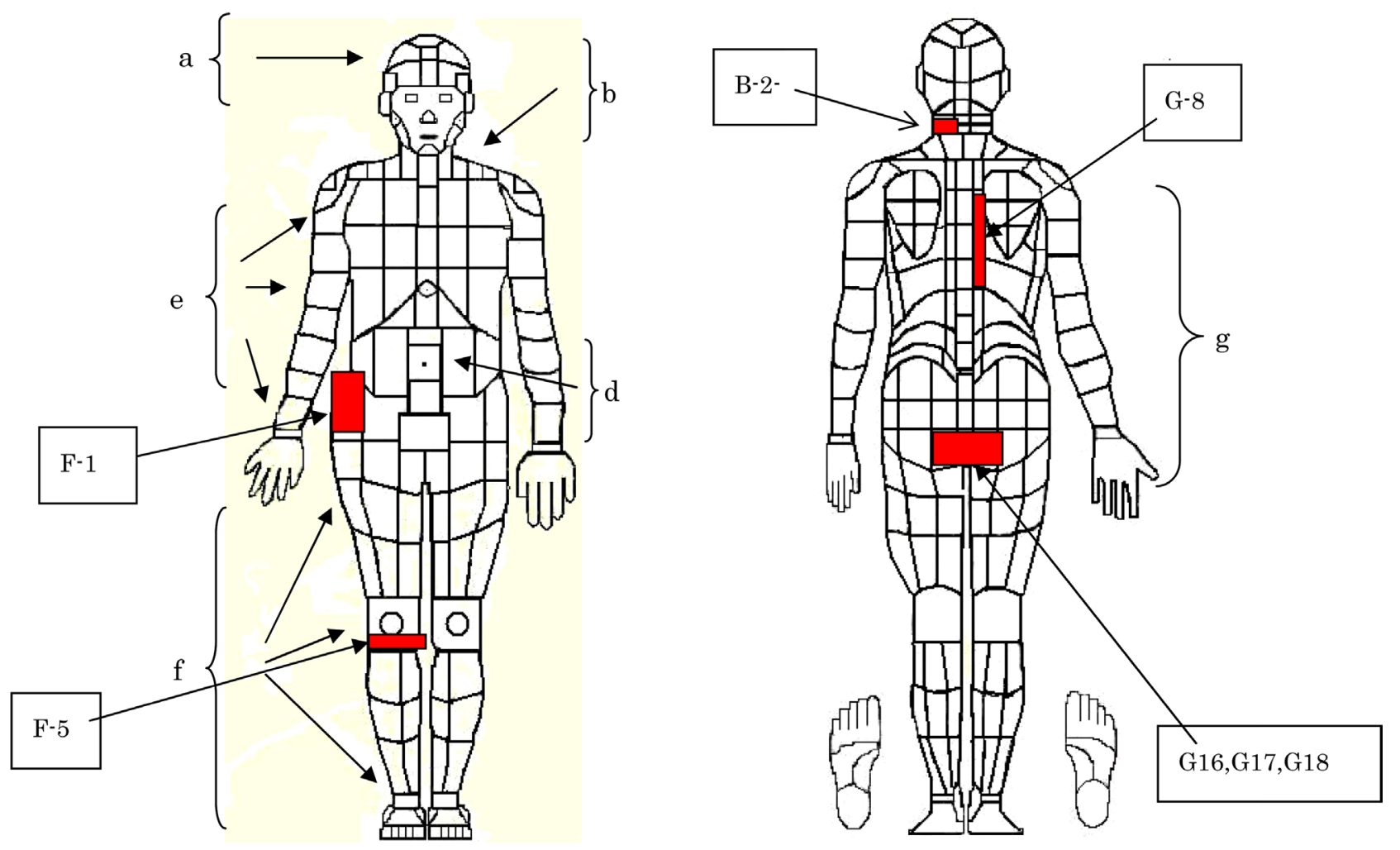

Figure 6. Partitioning of human body.

activate the malfunctioning area as identified in the previous section. For this purpose, we evaluate the painful and easily performed Active Motions through cross-correlation analysis typified by the Correlation Coefficient evaluation defined by Equation (3). Figure 8 shows the correlation coefficients between 21 "easy" Active Motions and 4 "painful" Active Motions, numbers 1, 2, 4 and 6. From Figure 8, we can easily identify Active Motions candidates that are suitable for remedying HMS parts causing pains.

We observe that there are still two or more candidates to remedy the pain, and in such cases we will apply other selection criteria by defining another criterion of optimization.

\section{Concluding Remarks}

The intention of this paper is to bridge the gap between conventional medicine and traditional therapy by removing obstacles and applying multidisciplinary approaches based on the systems engineering, and this has been attained to some degree. To demonstrate such an integrated approach, we have taken a therapist-guided exercise for restoring human musculoskeletal balance called the $S o$ matic Balance Restoration Therapy (SBRT). With this approach the practitioner has converted terminology used in therapy into wording of functional anatomical terms, and this effort has turned out to be useful for communication between the specialists of three different disciplines, namely, therapy, conventional medicine and systems engineering. Some examples have demonstrated a first step of 


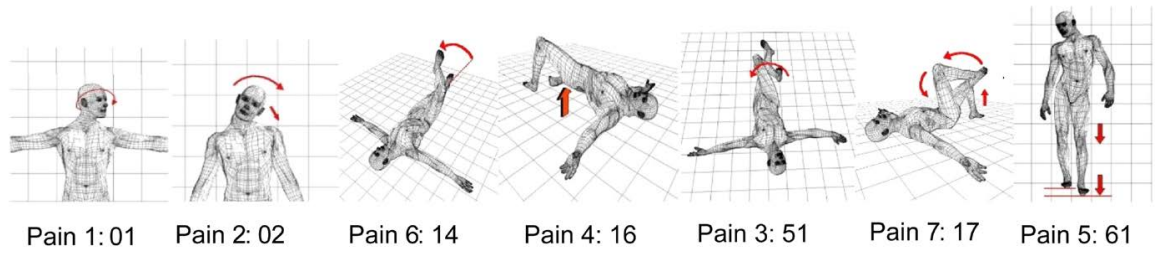

Figure 7. Seven active motions with pain in Table 3.

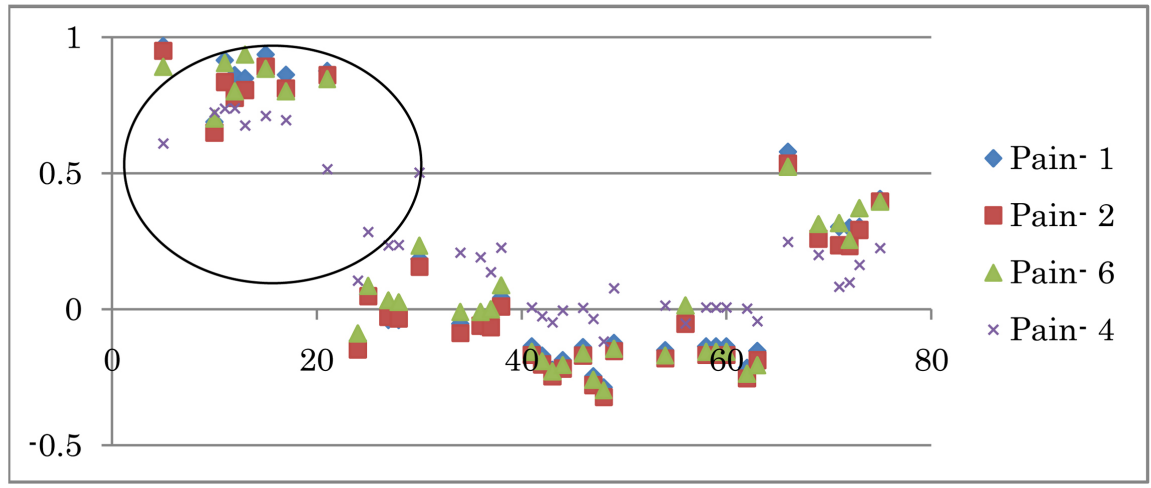

Active Motion No.

Figure 8. Correlation between active and painful motions shown in Table 4.

integrated and systematic approach for identifying malfunctions and remedying corrective exercise within the Fundamental Motion Elements.

\section{Acknowledgements}

The authors wish to express their appreciation to Dr. Motonaga of the Miyako-Jima Prefecture Hospital for his valuable advice.

\section{References}

[1] Hashimoto, K. (1981) Sotai Natural Exercise. Study Series. George Ohsawa Macrobiotic Foundation, Chico, CA, 26-34,

[2] Lars, A.-N. and Svensson, P. (2001) Referred Muscle Pain: Basic and Clinical Findings. Clinical Journal of Pain, 17, 11-19. https://doi.org/10.1097/00002508-200103000-00003

[3] Singh, S. and Ernst, E. (2008) Trick or Treatment: The Undeniable Facts about Alternative Medicine. WW Norton \& Company, New York.

[4] Ernst, E. and Canter, P.H. (2006) A Systematic Review of Systematic Reviews of Spinal Manipulation. Journal of the Royal Society of Medicine, 99, 192-196.

[5] Jonas, W.B. (1998) Alternative Medicine-Learning from the Past, Examining the Present, Advancing to the Future. JAMA, 280, 1616-1618.

https://doi.org/10.1001/jama.280.18.1616

[6] Bromley Milton, M., Börsbo, B., Rovner, G., Lundgren-Nilsson, Å., Stibrant-Sunnerhagen, K., et al. (2013) Is Pain Intensity Really That Important to Assess in Chronic Pain Patients? A Study Based on the Swedish Quality Registry for Pain Rehabilitation (SQRP). PLoS ONE, 8, e65483.

https://doi.org/10.1371/journal.pone.0065483 
[7] Hayden, J.A., Van Tulder, M.W. and Tomlinson, G. (2005) Systematic Review: Strategies for Using Exercise Therapy to Improve Outcomes in Chronic Low Back Pain. Annals of Internal Medicine, 142, 776-785. https://doi.org/10.7326/0003-4819-142-9-200505030-00014

[8] Laursen, T.M., Munk-Olsen, T. and Gasse, C. (2011) Chronic Somatic Comorbidity and Excess Mortality Due to Natural Causes in Persons with Schizophrenia or Bipolar Affective Disorder. PloS ONE, 6, e24597.

[9] Feine, J.S. and Lund, J.P. (1997) An Assessment of the Efficacy of Physical Therapy and Physical Modalities for the Control of Chronic Musculoskeletal Pain. Pain, 71, 5-23. https://doi.org/10.1016/S0304-3959(96)03287-3

[10] Floyd, R.T., et al. (1998) Manual of Structural Kinesiology. McGraw-Hill Higher Education, New York, 27-226.

[11] Herzog, W. (2000) Skeletal Muscle Mechanics from Mechanisms to Function. Wiley, 80-186.

[12] Zatsiorsky, V.M. (2002) Kinetics of Human Motion. 79-110.

[13] Kayo, M. and Ohkami, Y. (2013) Visualized Self-Exercise Therapy for Musculoskeletal Pain Alleviation. Science Journal of Medicine and Clinical Trials, 2013.

[14] Kayo, M. and Ohkami, Y. (2010) Structural Modeling of the Human Musculoskeletal System (HMS) for Clinical Treatment by Applying Joint-Connected Multibody Dynamics and System Approach. The International Mechanical Engineering Conference and Exhibition, Vancouver, 11-18 November 2010, Paper No. IMECE2010-40065.

[15] Kayo, M. and Ohkami, Y. (2009) A Method for Analyzing Fundamental Kinesiological Motions of Human Body by Applying Interpretive Structural Modeling (ISM). Presented at INCOSE IS09 in Singapore.

\section{Acronyms}

DOF Degrees-of-Freedom.

FME Fundamental Motion Elements.

HMS Human Musculoskeletal System.

SBRT Somatic Balance Restoration Therapy.

\section{Submit or recommend next manuscript to SCIRP and we will provide best service for you:}

Accepting pre-submission inquiries through Email, Facebook, LinkedIn, Twitter, etc.

A wide selection of journals (inclusive of 9 subjects, more than 200 journals)

Providing 24-hour high-quality service

User-friendly online submission system

Fair and swift peer-review system

Efficient typesetting and proofreading procedure

Display of the result of downloads and visits, as well as the number of cited articles

Maximum dissemination of your research work

Submit your manuscript at: http://papersubmission.scirp.org/

Or contact health@scirp.org 